\title{
Le NGF oriente les A-delta
}

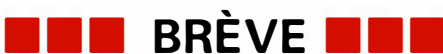

L'ototoxicité des aminosides est déterminée génétiquement. Les aminoglycosides ou aminosides (streptomycine, kanamycine, gentamicine, etc.) sont des antibiotiques largement utilisés ; ils sont toxiques pour le rein et pour l'oreille interne, et représentent une cause non négligeable de surdité. D. N. Hu et al. [1] (Shanghai, Chine) ont mené une étude épidémiologique dans un arrondissement de Shanghai ; 765 cas de surdimutité ont été identifiés dans une population de 483611 individus ; il s'agissait d'une surdité congénitale dans 285 cas et d'une surdité acquise dans 470, parmi lesquels 167 cas liés à l'administration d'aminosides prescrits dans l'enfance, avant tout la streptomycine. Dans ce dernier groupe, les auteurs ont identifié 47 cas où au moins un autre membre de la famille était également atteint d'une surdité due aux aminosides. Dans les cas familiaux d'ototoxicité, la durée du traitement était plus courte que dans les cas non familiaux. Il semble donc bien exister une certaine susceptibilité génétiquement déterminée à l'effet ototoxique des aminosides. Cela avait été déjà suggéré dans des travaux antérieurs. A partir de l'étude de 22 familles, les auteurs chinois suggèrent qu'il pourrait s'agir d'une transmission mitochondriale, seules les femmes transmettant la prédisposition. Une transmission autosomique dominante avait été avancée antérieurement. Cette susceptibilité familiale doit être connue des médecins prescripteurs, notamment chez l'enfant dont la mère a développé un accident d'ototoxicité.

[1. Hu DN, et al. J Med Genet 1991 ; $28: 79-83$.
Les facteurs trophiques agissent spécifiquement sur certaines populations neuronales au cours du développment.

Le nerve growth factor (NGF) [1] agit par exemple sur certains neurones des ganglions de la racine dorsale, responsables de la transmission des informations somatosensorielles (tactiles, nociceptives, etc.) depuis la périphérie jusqu'à la moelle épinière. Chez la souris, l'injection d'anticorps contre le NGF au cours du développement post-natal provoque ainsi une perte neuronale partielle dans ces ganglions [2] et une diminution parallèle du nombre des fibres somatosensorielles dites "primaires". L'équipe de Lorne Mendell (Stony Brook, NY, USA) a étudié les caractéristiques électrophysiologiques des neurones ainsi éliminés [3] afin de préciser leur identification. Le résultat s'est avéré plus complexe que prévu car les auteurs rapportent une action sélective du NGF sur un type de transmission bien plus que sur une population neuronale particulière. Les fibres somatosensorielles primaires se répartissent en trois groupes suivant leur vitesse de conduction (voir $\mathrm{m} / \mathrm{s} n^{\circ} 4$, vol. 5, p. 256): les fibres $\mathrm{C}$ convoient les influx à moins de 2 mètres par seconde, elles sont non myélinisées ; les fibres A, myélinisées, sont divisées en deux groupes, à conduction lente (fibres $A-\delta$ ) et à conduction rapide (fibres $A-\beta)$. Les fibres $A-\beta$ ne convoient que des informations non nociceptives ; au contraire, parmi les fibres $A-\delta$ et $C$, un certain contingent transmet des influx nés de stimulations nociceptives d'origine mécanique ou thermique. C'est la population de fibres $A-\delta$ qui transmet les informations nociceptives mécaniques (high threshold mechanoreceptor, HTMR) qui a attiré l'attention de l'équipe de Lorne Mendell. Normalement, $45 \%$ des fibres A- $\delta$ sont HTMR alors que $26 \%$ répondent à un léger mouvement des poils ( $D$-hair, DH). Après traitement de cinq semaines par anticorps anti-NGF, la proportion est totalement bouleversée avec $5 \%$ de HTMR et $72 \%$ de DH. Cette altération fonctionnelle, qui n'existe qu'après traitement au cours du développement, mais qui est alors permanente, découle d'une modification du type de récepteurs auquel sont liées les fibres et non à une perte sélective de neurones. Ce résultat est important car il donne une nouvelle dimension potentielle à l'action des facteurs neurotrophiques dans le système nerveux. On considérait jusqu'à présent que ces facteurs agissaient essentiellement, au cours du développement, comme agents de sélection permettant sélectivement à certains neurones de survivre. Le travail présenté ici suggère que, en plus de cet effet tout-ou-rien, le NGF jouerait un rôle important dans le choix fonctionnel de neurones survivants.

M. P.

1. Brachet P. Rôle du NGF dans la plasticité neuronale. médecine/sciences $1990 ; 6: 854-62$. 2. Hulsebosch CE, Perez-Polo JR, In vivo, anti-NGF induces sprouting of sensory axons in dorsal roots. Coggeshall RE. J Comp Neurol 1987 ; 259 : 445-51.

3. Ritter AM, Lewin GR, Kremer NE, Mendell LM. Requirement for nerve growth factor in the development of myelinated nociceptors in vivo. Nature $1991 ; 350$ : 500-2. 
La mutation du gène de la protéine $\beta$-amyloïde dans des formes familiales de maladie d'Alzheimer agit-elle au niveau traductionnel? médecine/sciences a récemment rendu compte de l'importante découverte d'une mutation dans le gène codant pour le précurseur de la protéine $\beta$-amyloïde dans des formes familiales de maladie d'Alzheimer. Le mécanisme proposé par les auteurs était celui d'une perturbation de l'ancrage à la membrane, pouvant sensibiliser le précurseur protéique à une coupure protéolytique anormale $\left(\mathrm{m} / \mathrm{s} n^{\circ} 3\right.$, vol. 7, p. 294). Dans une correspondance à Nature, E. Tanzi et T. Hyman [1] proposent maintenant une tout autre hypothèse. Ils notent que la région de l'ARN, siège de la mutation, peut adopter, normalement, une structure en tige-boucle ressemblant étrangement aux IRE (iron response element), éléments responsables de la régulation posttranscriptionnelle de la synthèse de la ferritine et du récepteur de la transferrine par le fer. Au niveau des messagers de la ferritine, ces éléments IRE se trouvent dans la région 5' non codante du messager et contrôlent leur traduction. Le mécanisme de cette régulation est la fixation sur l'élément IRE d'une protéine (IRE$\mathrm{BP}$ pour IRE-binding protein) qui se trouve dans un état oxydé, à faible affinité, en présence de fer, et réduit, à haute affinité, en l'absence de fer. La fixation de IRE-BP sur IRE, importante dans les conditions de carence martiale, bloque la synthèse de ferritine $\left(\mathrm{m} / \mathrm{s}^{\circ}\right.$ 8, vol. 4, p. 522) [2]. La mutation détectée dans les familles d'Alzheimer transforme un $G$ en $A$, créant un non-appariement au milieu de la tige de la structure tige-boucle de type IRE (figure 1 ). $\mathrm{Si}$ cette structure de la protéine $\beta$ amyloïde joue un rôle dans la traduction du messager, sa désorganisation doit être associée à l'absence de fixation de toute protéine la reconnaissant, et ainsi à la levée d'une éventuelle inhibition traductionnelle. Les conséquences de cette mutation pourraient ainsi être une hyperproduction du précurseur protéique de la pro- téine $\beta$-amyloïde secondaire à une augmentation de la traductibilité du messager. Le modèle de la régulation par le fer de l'abondance ou de la traductibilité des messagers de la ferritine ou du récepteur de la transferrine permet aussi d'évoquer que les éléments de type IRE présents dans le messager $\beta$-amyloïde pourraient eux-mêmes être la cible d'une régulation de la traduction ou de la stabilité du messager par des agents extérieurs tels que les métaux, notamment l'aluminium. Une telle éventualité expliquerait la modulation de la production de la protéine $\beta$ amyloïde par des facteurs de l'environnement, et par conséquent l'influence de ces facteurs sur la précocité d'apparition de signes de maladie d'Alzheimer.

G $\quad$ U
C A. A
A-C
G-G $\rightarrow A$
G-U
A-A
A-U
U-A C
G-C $1906 \quad 1931$

Figure 1. Structure en tige-boucle que pourrait prendre la séquence du messager du précurseur de la protéine $\beta$-amyloïde entre les nucléotides 1906 et 1931. La mutation $G \rightarrow A$ est représentée. La séquence de la boucle, CAGUGA, est similaire à celle des éléments IRE.

[1. Tanzi RE, Hyman BT. Nature 1991; 350 : 564.]

[2. Klausner RD, Harford JB. Science $1989 ; 246$ : 870-2.]

Protéine de liaison de l'immunosuppresseur SK 506 et protéine kinase C. La ciclosporine et le SK 506 sont de puissants inhibiteurs de l'activation des lympho- cytes $T$ qui se fixent sur des protéines de liaison dénommées, respectivement, cyclophiline et SKBP (SK 506 binding protein). Ces deux immunophilines ont une activité de peptidyl-prolyl-isomérase, ce qui signifie qu'elles peuvent induire l'isomérisation cis-trans de l'amide d'une proline à l'intérieur d'un peptide. Nous avons récemment indiqué $(\mathrm{m} / \mathrm{s}$ $n^{\circ} 10$, vol. $6, p$. 1022) que cette activité enzymatique est puissamment inhibée par la fixation des ligands spécifiques de ces immunophilines. Le pouvoir immunosuppresseur n'est cependant pas dû à cette inhibition puisque des fragments dérivés du SK 506 sont de bons ligands de la SKBP, inhibent l'activité de peptidylprolyl-isomérase, mais ne sont pas des immunosuppresseurs. Dans une lettre à la revue Cell [1], Goeb] (Indianapolis, IN, USA) vient de montrer que la SKBP est probablement un inhibiteur de la protéine kinase $\mathrm{C}$ d'un poids moléculaire de 12 000, précédemment décrit [2]. La protéine kinase $\mathrm{C}$ joue un rôle essentiel dans l'activation des lymphocytes $T$, et l'on conçoit que tout inhibiteur de cette enzyme puisse être un puissant immunosuppresseur. Le SK 506 prévient l'activation de cellules T clonées par les esters de phorbol, ligands et activateurs puissants de la protéine kinase C. Le SK 506 pourrait donc moduler l'activité inhibitrice de la SKBP de $12 \mathrm{kDa}$, par exemple en facilitant son interaction avec la protéine kinase C. Par extension, on peut suggérer que la ciclosporine agirait par un mécanisme similaire. Si ces résultats sont confirmés, ils constituent un pas décisif dans la compréhension du mécanisme d'action des immunosuppresseurs de cette famille. Ils pourraient aussi déboucher sur l'identification de modulateurs très spécifiques de la protéine kinase $\mathrm{C}$ qui permettront d'étudier plus facilement les multiples fonctions de cette enzyme.

[1. Goebl MG, et al. Cell 1991; 64 1051-2.]

[2. Mozier M, et al. Eur J Biochem $1990 ; 194$ : 19-23.] 